Hydrodynamic, Atomic Kinetic, and Monte Carlo Radiation Transfer Models of the X-ray Spectra of Compact Binaries

C. W. Mauche, D. A. Liedahl, S. Akiyama, T. Plewa

February 11,2008 
This document was prepared as an account of work sponsored by an agency of the United States government. Neither the United States government nor Lawrence Livermore National Security, LLC, nor any of their employees makes any warranty, expressed or implied, or assumes any legal liability or responsibility for the accuracy, completeness, or usefulness of any information, apparatus, product, or process disclosed, or represents that its use would not infringe privately owned rights. Reference herein to any specific commercial product, process, or service by trade name, trademark, manufacturer, or otherwise does not necessarily constitute or imply its endorsement, recommendation, or favoring by the United States government or Lawrence Livermore National Security, LLC. The views and opinions of authors expressed herein do not necessarily state or reflect those of the United States government or Lawrence Livermore National Security, LLC, and shall not be used for advertising or product endorsement purposes.

This work performed under the auspices of the U.S. Department of Energy by Lawrence Livermore National Laboratory under Contract DE-AC52-07NA27344. 


\title{
Hydrodynamic, Atomic Kinetic, and Monte Carlo Radiation Transfer Models of the X-ray Spectra of Compact Binaries
}

\author{
C. W. Mauche, D. A. Liedahl, S. Akiyama (Lawrence Livermore National \\ Laboratory), and T. Plewa (University of Chicago)
}

\begin{abstract}
We describe the results of an effort, funded by the Lawrence Livermore National Laboratory Directed Research and Development Program, to model, using FLASH time-dependent adaptive-mesh hydrodynamic simulations, XSTAR photoionization calculations, HULLAC atomic data, and Monte Carlo radiation transport, the radiatively-driven photoionized wind and accretion flow of high-mass X-ray binaries (HMXBs). In this final report, we describe the purpose, approach, and technical accomplishments of this effort, including maps of the density, temperature, velocity, ionization parameter, and emissivity distributions of the X-ray emission lines of the well-studied HMXB Vela X-1.
\end{abstract}

\section{$\S 1$. Introduction}

As described by Castor, Abbott, and Klein (hereafter CAK), ${ }^{1)}$ mass loss in the form of a high velocity wind is driven from the surface of an OB star by radiation pressure on a multitude of resonance transitions of intermediate charge states of cosmically abundant elements. The wind is characterized by a mass-loss rate $\dot{M} \sim$ $10^{-6}-10^{-5} \mathrm{M}_{\odot} \mathrm{yr}^{-1}$ and a velocity profile $V(R) \sim V_{\infty}\left(1-R_{\mathrm{OB}} / R\right)^{\beta}$, where $\beta \approx \frac{1}{2}$, the terminal velocity $V_{\infty} \sim 3 V_{\mathrm{esc}}=3\left(2 G M_{\mathrm{OB}} / R_{\mathrm{OB}}\right)^{1 / 2} \sim 1500 \mathrm{~km} \mathrm{~s}^{-1}, R$ is the distance from the $\mathrm{OB}$ star, and $M_{\mathrm{OB}}$ and $R_{\mathrm{OB}}$ are respectively the mass and radius of the OB star. In a detached high-mass X-ray binary (HMXB), a compact object, typically a neutron star, captures a fraction $f \sim \pi R_{\mathrm{BH}}^{2} / 4 \pi a^{2}$ of the OB star wind, where $a$ is the binary separation, $R_{\mathrm{BH}}=2 G M_{\mathrm{NS}} /\left[V(a)^{2}+c_{\mathrm{s}}^{2}\right]$ is the Bondi-Hoyle radius, $c_{\mathrm{S}} \sim 10\left(T / 10^{4}\right)^{1 / 2} \mathrm{~km} \mathrm{~s}^{-1}$ is the sound speed, and $T$ is the wind temperature. Accretion of this material onto the neutron star powers an X-ray luminosity $L_{\mathrm{X}} \sim$ $f G \dot{M} M_{\mathrm{NS}} / R_{\mathrm{NS}} \sim 10^{36}-10^{37} \mathrm{erg} \mathrm{s}^{-1}$, where $M_{\mathrm{NS}}$ and $R_{\mathrm{NS}}$ are respectively the mass and radius of the neutron star. The resulting X-ray flux photoionizes the wind and reduces its ability to be radiatively driven, both because the higher ionization state of the plasma results is a reduction in the number of resonance transitions, and because the energy of the transitions shifts to shorter wavelengths where the overlap with the stellar continuum is lower. To first order, the lower radiative driving results in a reduced wind velocity near the neutron star $V(a)$, which increases the BondiHoyle radius $R_{\mathrm{BH}}$, which increases the accretion efficiency $f$, which increases the X-ray luminosity $L_{\mathrm{X}}$. In this way, the X-ray emission of HMXBs is the result of a complex interplay between the radiative driving of the wind of the OB star and the photoionization of the wind by the neutron star.

Known since the early days of X-ray astronomy, HMXBs have been extensively studied observationally, theoretically, ${ }^{2)-4)}$ and computationally. ${ }^{5)-8)}$ They are excellent targets for X-ray spectroscopic observations because the large covering fraction of 
the wind and the moderate X-ray luminosities result in large volumes of photoionized plasma that produce strong recombination lines and narrow radiative recombination continua of H- and He-like ions, as well as fluorescent lines from lower charge states.

\section{§2. Vela X-1}

Vela X-1 is the prototypical detached HMXB, having been studied extensively in nearly every waveband, particularly in X-rays, since its discovery as an X-ray source during a rocket flight four decades ago. It consists of a B0.5 Ib supergiant and a magnetic neutron star in an 8.964-day orbit. From an X-ray spectroscopic point of view, Vela X-1 distinguished itself in 1994 when Nagase et al., ${ }^{9)}$ using $A S C A$ SIS data, showed that, in addition to the well-known $6.4 \mathrm{keV}$ emission line, the eclipse $\mathrm{X}$-ray spectrum is dominated by recombination lines and continua of $\mathrm{H}$ - and He-like $\mathrm{Ne}, \mathrm{Mg}, \mathrm{Si}, \mathrm{S}, \mathrm{Ar}$, and Fe. These data were subsequently modeled in detail by Sako et al., ${ }^{10)}$ using a kinematic model in which the photoionized wind was characterized by the ionization parameter $\xi \equiv L_{\mathrm{X}} / n r^{2}$, where $r$ is the distance from the neutron star and $n$ is the number density, given by the mass-loss rate and velocity law of an undisturbed CAK wind. Vela X-1 was subsequently observed with the High Energy Transmission Grating (HETG) onboard the Chandra X-ray Observatory in 2000 for $30 \mathrm{ks}$ in eclipse ${ }^{11)}$ and in 2001 for 85, 30, and $30 \mathrm{ks}$ in eclipse and at binary phases 0.25 and 0.5 , respectively. ${ }^{12), 13)}$ Watanabe et al., ${ }^{13)}$ using very similar assumptions as Sako et al. and a Monte Carlo radiation transfer code, produced a global model of Vela X-1 that simultaneously fit the HETG spectra from the three binary phases with a wind mass-loss rate $\dot{M} \approx 2 \times 10^{-6} \mathrm{M}_{\odot} \mathrm{yr}^{-1}$ and terminal velocity $V_{\infty}=1100 \mathrm{~km} \mathrm{~s}^{-1}$. One of the failures of this model was the velocity shifts of the emission lines between eclipse and phase 0.5 , which were observed to be $\Delta V \approx 400-500 \mathrm{~km} \mathrm{~s}^{-1}$, while the model simulations predicted $\Delta V \sim 1000 \mathrm{~km} \mathrm{~s}^{-1}$. In order to resolve this discrepancy, Watanabe et al. performed a $1 \mathrm{D}$ calculation to estimate the wind velocity profile along the line of centers between the two stars, accounting, in an approximate way, for the reduction of the radiative driving due to photoionization. They found that the velocity of the wind near the neutron star is lower by a factor of 2-3 relative to an undisturbed CAK wind, which was sufficient to explain the observations. However, these results were not fed back into their global model to determine the effect on the X-ray spectrum.

\section{§3. Hydrodynamic Simulations}

To make additional progress in our understanding of the wind and accretion flow of HMXBs in general and Vela X-1 in particular - to bridge the gap between the detailed hydrodynamic models of Blondin et al. and the simple kinetic-spectral models of Sako et al. and Watanabe et al. - we undertook in our LDRD project to develop improved models of radiatively-driven photoionized accretion flows, with the goal of producing synthetic X-ray spectral models that possess a level of detail commensurate with the grating spectra returned by Chandra and XMM-Newton.

This project combines (1) XSTAR ${ }^{14)}$ photoionization calculations, (2) HUL- 
LAC ${ }^{15)}$ emission models appropriate to X-ray photoionized plasmas, (3) improved models of the radiative driving of the photoionized wind, (4) FLASH ${ }^{16}$ ) two- and three-dimensional time-dependent adaptive-mesh hydrodynamics simulations, and (5) a Monte Carlo radiation transport code. ${ }^{17}$ ) Radiative driving of the wind is accounted for via the force multiplier formalism, ${ }^{1)}$ accounting for X-ray photoionization and non-LTE population kinetics using HULLAC atomic data for $2 \times 10^{6}$ lines of 35,000 energy levels of 166 ions of the 13 most cosmically abundant elements. In addition to the usual hydrodynamic quantities, the FLASH calculations account for (a) the gravity of the OB star and neutron star, (b) Coriolis and centrifugal forces, (c) radiative driving of the wind as a function of the local ionization parameter, temperature, and optical depth, $(d)$ photoionization and Compton heating of the irradiated wind, and $(e)$ radiative cooling of the irradiated wind and the "shadow wind" behind the OB star.
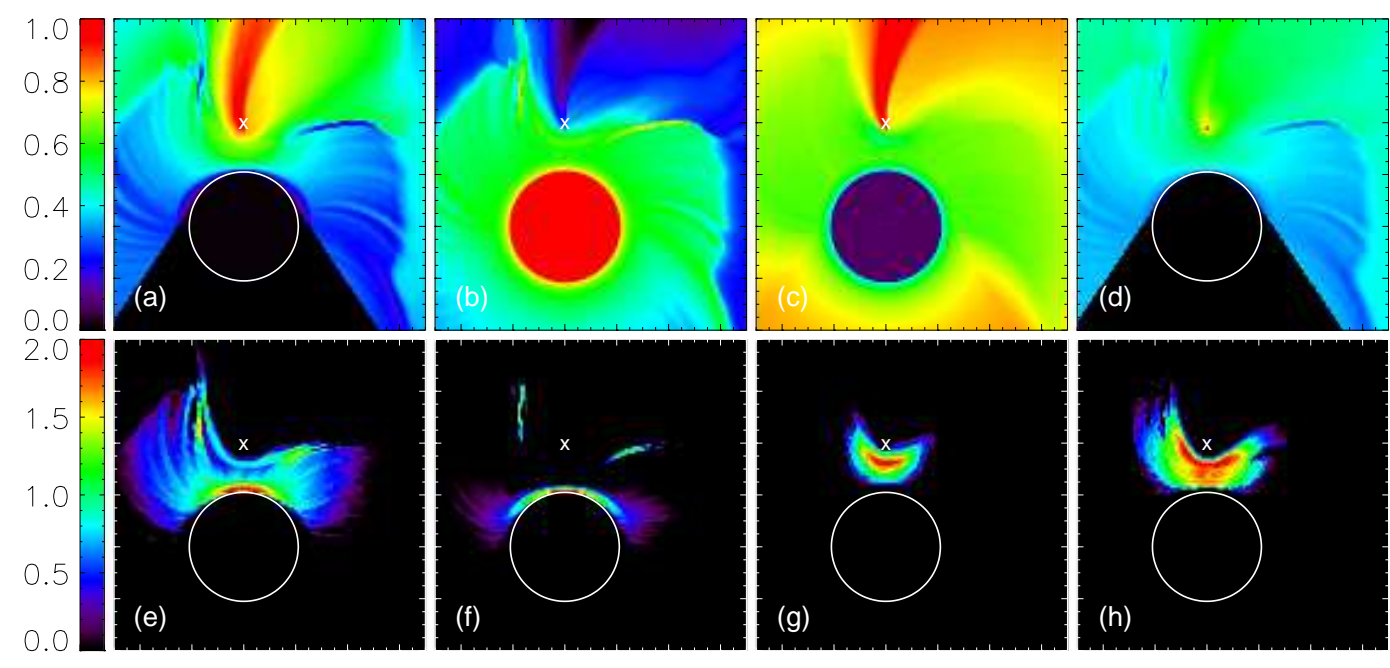

Fig. 1. Top: Color-coded maps of $(a) \log T(\mathrm{~K})=[4.4,8.3],(b) \log n\left(\mathrm{~cm}^{-3}\right)=[7.4,10.8],(c)$ $\log v\left(\mathrm{~km} \mathrm{~s}^{-1}\right)=[1.3,3.5]$, and $(d) \log \xi\left(\mathrm{erg} \mathrm{cm} \mathrm{s}^{-1}\right)=[1.1,7.7]$ in the orbital plane of Vela X1. The positions of the B star and neutron star are shown by the circle and the "x," respectively. The horizontal axis $x=[-0.5,0.7] \times 10^{13} \mathrm{~cm}$, and the vertical axis $y=[-0.4,0.8] \times 10^{13} \mathrm{~cm}$. Bottom: Corresponding maps of the log of the emissivity of $(e)$ Si XIV Ly $\alpha,(f)$ Si XIII He $\alpha$, $(g)$ FeXXVI Ly $\alpha$, and $(h)$ Fe XXV He $\alpha$. In each case, two orders of magnitude are plotted.

\section{$\S 4$. 2D Simulations}

To demonstrate typical results of our simulations, we show in the upper panel of Fig. 1 color-coded maps of the $\log$ of the $(a)$ temperature $T,(b)$ density $n,(c)$ velocity $v$, and $(d)$ ionization parameter $\xi$ of a FLASH simulation with parameters appropriate to Vela X-1. This is a $2 \mathrm{D}$ simulation in the binary orbital plane, has a resolution of $\Delta l=9.4 \times 10^{10} \mathrm{~cm}$, and, at the time step shown $(t=100 \mathrm{ks})$, the 
relatively slow $\left(V \approx 400 \mathrm{~km} \mathrm{~s}^{-1}\right)^{*)}$ irradiated wind has reached just $\sim 2$ stellar radii from the stellar surface. The various panels show (1) the effect of the Coriolis and centrifugal forces, which cause the flow to curve clockwise, (2) the cool, fast wind behind the OB star, (3) the hot, slow irradiated wind, (4) the hot, low density, high velocity flow downstream of the neutron star, and (5) the bow shock and two flanking shocks formed where the irradiated wind collides with the hot disturbed flow in front and downstream of the neutron star.

Given these maps, it is straightforward to determine where in the binary the $\mathrm{X}$-ray emission originates. To demonstrate this, we show in the lower panel of Fig. 1 color-coded maps of the log of the emissivity of $(e)$ Si XIV Ly $\alpha,(f)$ Si XIII He $\alpha,(g)$ Fe XXVI Ly $\alpha$, and $(h)$ FeXXV He $\alpha$. The gross properties of these maps agree with Fig. 24 of Watanabe et al., but they are now (1) quantitative rather than qualitative and (2) specific to individual transitions of individual ions. The maps also capture features that otherwise would not have been supposed, such as the excess emission in the H- and He-like Si lines downstream of the flanking shocks. Combining these maps with the velocity map (Fig. 1c), these models make very specific predictions about (1) the intensity of the emission features, (2) where the emission features originate, and (3) their velocity widths and amplitudes as a function of binary phase.

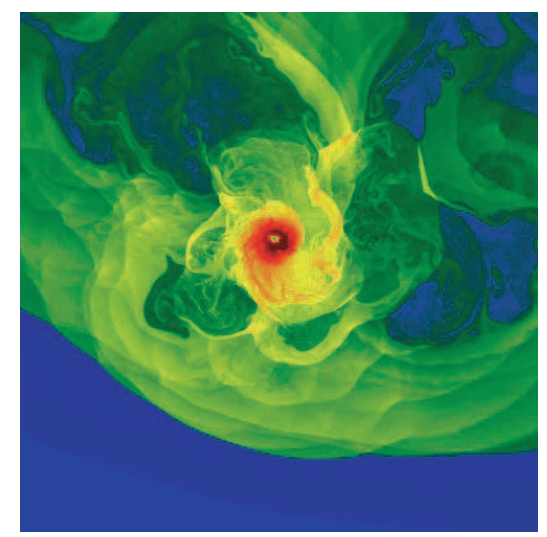

Fig. 2. Color-coded map of $\log n\left(\mathrm{~cm}^{-3}\right)=[8.8,10.8]$ (blue to red) for a 2D FLASH simulation of Vela X-1, showing the flow in the immediate vicinity of the neutron star $\left(\Delta x=\Delta y=5 \times 10^{10}\right.$ $\mathrm{cm})$. The OB star wind is coming in from the lower left and the low-density pockets in the down-stream flow are filled with $\log T(\mathrm{~K}) \approx 8.5$ plasma.

As an aside, we wish to point out the power and importance of the adaptive mesh refinement capability in FLASH. Toward this end, we show in Figure 2 a closeup view of the hydrodynamic flow calculated by FLASH in the immediate vicinity of the neutron star. This frame, which shows a color-coded map of the log of the density, is $5 \times 10^{10} \mathrm{~cm}$ on a side and is taken from a simulation spanning the full scale of the binary: $\Delta x=1.2 \times 10^{13} \mathrm{~cm}$ by $\Delta y=1.6 \times 10^{13} \mathrm{~cm}$. The 15 levels of refinement in this simulation allows us to resolve the flow down to $\Delta l=3.7 \times 10^{8}$ $\mathrm{cm}$, and so follow the complex interaction of the OB star wind with the neutron star,

*) Note that this velocity reproduces the value that Watanabe et al. found was needed to match the velocity of the emission lines in the Chandra HETG spectra of Vela X-1. 
including the formation and disruption of the accretion disk around the neutron star, which leads to variations in the X-ray luminosity, and the formation and growth of instabilities between the fast upstream and the hot down-stream flow, which produce flow irregularities in the accretion wake on the scale of the binary.
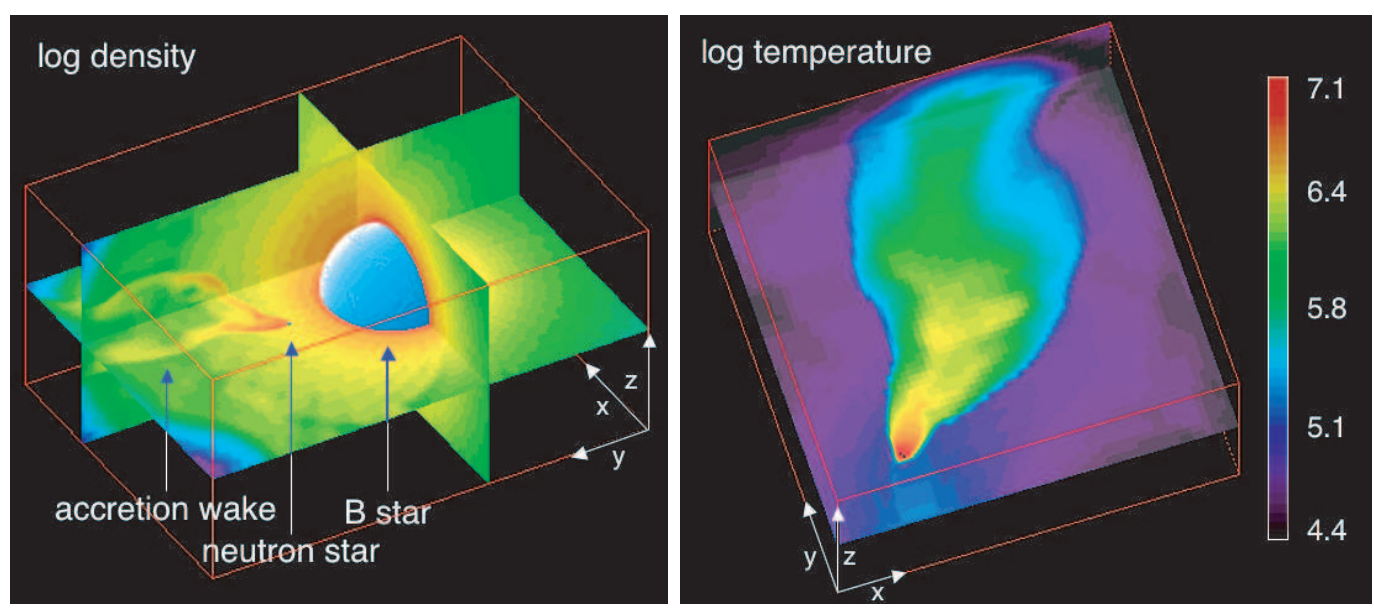

Fig. 3. Color-coded slices of $\log n$ (left) and $\log T$ (right) for a 3D FLASH simulation of Vela X-1, showing the OB star, neutron star, and accretion wake (shown in close-up in the left panel).

\section{$\S 5$. 3D Simulations and Synthetic X-ray Spectra}

Having developed and extensively tested our software on various 2D simulations, we next moved to a more limited run of full 3D simulations of an HMXB with parameters appropriate to Vela X-1. Figure 3 shows two frames from one of these simulations, showing in the left panel the log of the number density for three orthogonal slices through the $3 \mathrm{D}$ computational volume on the full scale of the binary $\left(\Delta x=1.2 \times 10^{13} \mathrm{~cm}, \Delta y=1.6 \times 10^{13} \mathrm{~cm}\right.$, and $\left.\Delta z=0.8 \times 10^{13} \mathrm{~cm}\right)$ and in the right panel the log of the temperature for the accretion wake behind the neutron star.

In the next step in the process, we performed Monte Carlo radiation transfer calculations of photons emitted near the neutron star, which interact in the computational volume, acquiring spectral features, before escaping to infinity. The Monte Carlo code used to perform these calculations was developed by members of our team (Mauche and Liedahl) as part of a previous LDRD project ("Structure and Spectroscopy of Black Hole Accretion Disks;" 02-ERD-004) which combined detailed computer models of accretion disk atmospheres with Monte Carlo radiation transfer calculations to elucidating the high radiation density environments associated with mass flows in the curved spacetime near gravitationally collapsed objects. As described by Mauche et al., ${ }^{17)}$ the Monte Carlo code accounts for Compton and photoelectric opacity for 446 subshells of 140 ions of the 12 most abundant elements. Following photoabsorption by K-shell ions, we generate radiative recombination continua (RRC) and recombination line cascades in a probabilistic manner using the recombination cascade calculations described by Sako et al. ${ }^{10)}$ The shapes of the 
RRCs are determined by the functional form of the photoionization cross sections and the local electron temperature. Recombination emission from L-shell ions is ignored, since it is suppressed by resonant Auger destruction. ${ }^{18)}$

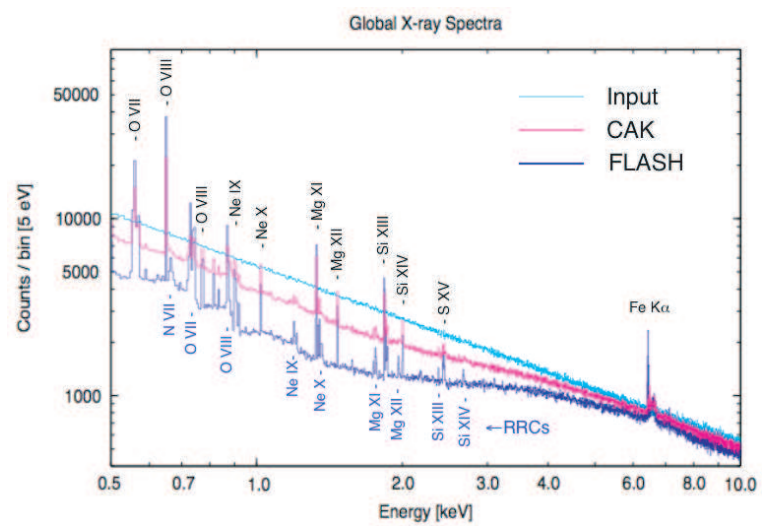

Fig. 4. Monte Carlo radiation transfer simulations of the $0.5-10 \mathrm{keV}$ X-ray spectrum of Vela X-1 for an undisturbed CAK-type flow (pink) and our detailed 3D FLASH hydrodynamic simulation (dark blue). Strong emission lines are labeled in black and strong radiative recombination continua (RRCs) are labeled in dark blue.

Two examples of the synthetic X-ray spectra from such Monte Carlo calculations are shown in Figure 4. The upper light-blue line is the assumed (featureless) power law spectrum emitted by the neutron star, while the spectra escaping the computational volume, with the rich set of photoelectric absorption edges, RRCs and recombination line spectral features, are shown in pink and dark blue for, respectively, an undisturbed CAK-type flow, as assumed by Sako et al. ${ }^{10)}$ and Watanabe et al. ${ }^{13)}$ and our detailed 3D FLASH hydrodynamic simulation. This figure demonstrates the significant differences in the synthetic spectra for the simple CAK and our detailed hydrodynamic model, highlighting the importance of the details of the 3D model to the emitted spectra. The next step in this process is a careful comparison of these synthetic spectra to the grating spectra of Vela X-1 returned by Chandra and XMM-Newton. In this way, it will be possible to constrain various parameters of Vela $\mathrm{X}-1$, such as the OB star wind mass-loss rate, velocity profile, terminal velocity, and elemental abundances. For the mass-loss rate alone, there is a factor of 10 discrepancy between the estimates provided from ultraviolet and X-ray observations, ${ }^{10}$ ) and a similar level of discrepancy between the X-ray work of Sako et al. ${ }^{10)}$ and Watanabe et al. ${ }^{13)}$ Knowledge of the wind mass-loss rate bears on such basic questions as the long-term evolution of the binary and the chemical enrichment of the interstellar medium.

\section{$\S 6 . \quad$ Exit Plan}

Based on the good and promising progress of the research summarized above, we wrote, submitted, and had approved in 2007 a NASA Astrophysics Theory Program research proposal entitled "Hydrodynamical and Spectral Simulations of X-ray 
Irradiated Stellar Winds." Funds for this two-year project amount to approximately $\$ 300 \mathrm{~K}$, nominally sufficient to pay a postdoc's salary for two years, plus travel and publication costs. In addition, we wrote, submitted, but did not have approved a NASA Chandra Guest Observer Program proposal entitled "X-ray Tomography of the High-Mass X-ray Binary Vela X-1," in which we proposed a long (630 kiloseconds) Chandra HETG observation of Vela X-1. The budgets for this program are not determined until after the proposals are accepted, but they are approximately $\$ 130 \mathrm{~K}$ for such long proposed observations. We plan to use the input from the peer reviewers to rework this proposal and resubmit it in March of this year. In summary, we are and plan to continue the research begun under our LDRD, and have access to work-for-others (primarily, NASA) funds which with to pay for these activities.

\section{Acknowledgments}

This work performed under the auspices of the U.S. Department of Energy by Lawrence Livermore National Laboratory under Contract DE-AC52-07NA27344. This work was funded by the Laboratory Directed Research and Development Program at LLNL under project tracking code 05-ERD-044. T. Plewa's contribution to this work was supported in part by the U.S. Department of Energy under Grant No. B523820 to the Center for Astrophysical Thermonuclear Flashes at the University of Chicago. The FLASH software used in this work was developed in part by the DOE-supported ASC/Alliance Center for Astrophysical Thermonuclear Flashes at the University of Chicago.

\section{References}

1) J. I. Castor, D. C. Abbott, and R. I. Klein, Ap.J. 195 (1975), 157, CAK.

2) S. Hatchett and R. McCray, Ap.J. 211 (1977), 552.

3) R. McCray, T. R. Kallman, J. I. Castor, and G. L. Olson, Ap.J. 282 (1984), 245.

4) I. R. Stevens and T. R. Kallman, Ap.J. 365 (1990), 321.

5) J. M. Blondin, T. R. Kallman, B. A. Fryxell, and R. E. Taam, Ap.J. 356 (1990), 591.

6) J. M. Blondin, I. R. Stevens, and T. R. Kallman, Ap.J. 371 (1991), 684.

7) J. M. Blondin, Ap.J. 435 (1994), 756.

8) J. M. Blondin and J. W. Woo, Ap.J. 445 (1995), 889.

9) F. Nagase, G. Zylstra, T. Sonobe, T. Kotani, H. Inoue, and J. Woo, Ap.J. 436 (1994), L1.

10) M. Sako, D. A. Liedahl, S. M. Kahn, and F. Paerels, Ap.J. 525 (1999), 921.

11) N. S. Schulz, C. R. Canizares, J. C. Lee, and M. Sako, Ap.J. 564 (2002), L21.

$12)$ G. Goldstein, D. P. Huenemoerder, and D. Blank, A.J. 127 (2004), 2310.

13) S. Watanabe, et al., Ap.J. 651 (2006), 421.

14) T. Kallman and M. Bautista, Ap.J.S. 133 (2001), 221

15) A. Bar-Shalom, M. Klapisch, and J. Oreg, Phys. Rev. A38 (1988), 1773

16) B. Fryxell, et al., Ap.J.S. 131 (2000), 273.

17) C. W. Mauche, D. A. Liedahl, B. F. Mathiesen, M. A. Jimenez-Garate, and J. C. Raymond, Ap.J. 606 (2004), 168.

18) R. R. Ross, A. C. Fabian, \& W. N. Brandt, M.N.R.A.S. 278 (1996), 1082 